\title{
Clinical Characteristics and Gustatory Profiles in Patients with Subjective Taste Complaints
}

\author{
Seo-Yeong Kim, Jin-Seok Byun, Jae-Kwang Jung, Jae-Kap Choi \\ Department of Oral Medicine, School of Dentistry, Kyungpook National University, Daegu, Korea
}

Received August 14, 2019

Revised August 26, 2019

Accepted August 26, 2019
Correspondence to:

Jae-Kap Choi

Department of Oral Medicine, School of

Dentistry, Kyungpook National University,

2177 Dalgubeol-daero, Jung-gu, Daegu

41940, Korea

Tel: +82-53-600-7321

Fax: +82-53-426-2195

E-mail:jhchoi@knu.ac.kr

https://orcid.org/0000-0001-6773-7507
Purpose: Patients with taste complaints presenting with various abnormal perceptions and alterations in gustatory function are often encountered in dental clinics. Since taste perception is thought to be influenced by numerous factors including neurological and psychological factors, the gustatory profiles of patients complaining of taste abnormalities should be very different. However, the gustatory profiles based on the clinical subtypes of taste complaints have not been fully studied. This study aimed to better understand the gustatory profiles depending on the clinical subtypes of taste complaints.

Methods: Clinical data from 169 patients with complaints of altered taste were retrospectively collected to analyse their clinical and gustatory profiles. These complaints were subdivided into hypergeusia, hypogeusia, and dysgeusia for each taste quality according to the clinical types of these complaints. The gustatory profiles were then established by analysing the detection and recognition thresholds for each taste quality depending on the clinical subtypes of taste complaints.

Results: Clinical analysis revealed that patients with taste complaints had widely diverse clinical profiles. There were significant differences between males and females with taste complaints in the prevalence rates of symptoms like dry mouth, tongue coating, and burning sensation. While hypogeusia (76.3\%) was the most frequent type of taste complaint, it was revealed that the taste thresholds were not always consistent with the patient's description of gustatory symptoms.

Conclusions: Patients with taste complaints exhibited diverse clinical profiles with sex differences. Considering the diversity of the taste complaints, the quantitative gustatory testing methods can be valuable to differentially evaluate the presence and intensity of altered taste in patients with these complaints.

Key Words: Clinical study; Epidemiology; Taste disorder; Taste threshold

\section{INTRODUCTION}

The gustatory sensation is one of the special fundamental sensory systems which play a pivotal role in determining the quality of life by making it possible to predict the danger of poisoned or rotten foods and to provide the pleasure of food intake [1]. The chemical stimuli of tastants were mediated through electrical signals of neural impulse by the taste buds located in the oral epithelium on the tongue, palate, and the posterior wall of the pharynx. This signalling information travels via the brainstem, where other sensations are possibly integrated through multi-synaptic processing and then transmitted to the cerebral cortex where the information from each neural impulse gets organized to determine the gustatory perception [2].

Taste complaints that are often encountered in the dental

Copyright (c) 2019 Korean Academy of Orofacial Pain and Oral Medicine. All rights reserved.

(c) This is an open-access article distributed under the terms of the Creative Commons Attribution Non-Commercial License (http://creativecommons.org/licenses/by-nc/4.0/), which permits unrestricted non-commercial use, distribution, and reproduction in any medium, provided the original work is properly cited. 
clinics present with various abnormal alterations in gustatory perception. The gustatory alterations could be perceived due to pathological disturbances at any steps of sensation, integration, or organization [2]. The gustatory sensations could be affected by the delivery conditions of the gustatory components, as well as the integrity of the taste bud cells and the underlying nerves. The former might frequently be observed in patients with hyposalivation, tongue biofilm, and oral inflammation. In addition, it was reported that taste complaints were initiated by a variety of systemic drugs and diseases [3]. The mechanistic causes of gustatory dysfunction were classified into transport, sensory, and neuronal problems [4]. It was also known that sensory inputs like olfactory, tactile, and thermal stimuli were intimately involved in gustatory perception [2]. Since it is not easy to discriminate the sensation disturbances from the perception disturbances, the terms of gustatory (taste) dysfunction, gustatory (taste) disorder, and gustatory (taste) complaints have been interchangeably used [5]. However, considering that the subjective taste complaints could be caused by olfactory or somatosensory disorder/dysfunction or psychological problems without any abnormal findings of gustatory sensitivity, taste complaints appear to be a more suitable term encompassing all the conditions related with the selfreported taste alterations.

The prevalence rate of taste disorders was known to range from $0.6 \%$ to $5.3 \%$ among various populations [68]. Recently, an epidemiological study conducted through population-based surveys reported that the prevalence of self-reported taste alterations amounted to 19\% and progressively increased with age [9]. However, several studies have indicated that among the patients with subjective complaints of taste loss, only a minority demonstrated a measurable loss in the quantitative taste tests, thereby exhibiting the clinical importance of quantitative gustatory analysis [10,11]. Various methods that can be categorized as chemogustometry and electrogustometry, have been introduced for the quantitative assessment of taste. Most taste clinics have adopted one of the quantitative methods for better evaluation of taste disorders [12].

Few studies have reported the differential features of gustatory outcomes according to the clinical types of taste complaints. In addition, there were no detailed studies examining the differences in the clinical profiles of patients with taste complaints according to sex.

Therefore, this study aimed to determine the sex differences in the clinical profiles and to investigate the differential pattern of quantitative gustatory outcomes according to the clinical types of taste complaints. For this purpose, the study was retrospectively conducted to analyse the clinical characteristics of patients with taste disorders and to compare the quantitative outcome of gustatory tests according to the clinical types of taste complaints.

\section{MATERIALS AND METHODS}

This study was based on the data obtained from hospital records of the patients who visited the Department of Oral Medicine, Kyungpook National University Dental Hospital from 2003 to 2019 with the chief complaint of taste problems. After excluding patients who did not undergo gustometric tests or had insufficient records, 169 patients were included in the study. The study protocol was approved by the Institutional Review Board of Kyungpook National University Dental Hospital (IRB no. KNUDH-2019-01-05-00). Acquisition of informed consent was exempted by the Board.

Demographic and clinical information included sex, age, gustatory complaints, past medical/dental history, salivary flow, gustatory thresholds, intraoral findings, such as tongue coating, oral malodour, etc. Taste complaints were broadly classified into hypergeusia, hypogeusia, and dysgeusia and then according to the quality of the affected taste, they were further subclassified into sweet, salty, bitter, and sour. Systemic conditions were evaluated using a questionnaire and systemic review. The chief complaints were analysed in detail with regard to the onset, progression, severity of taste problem, accompanied symptoms, suspicious causes, prior treatment and response, and the accompaniment of olfactory problems. Physical and radiographic examinations were carried out in order to identify the presence of oral dryness, viral/bacterial/fungal infections, and oral mucosal lesions. For the clinical evaluation of oral dryness, the flow rate of unstimulated whole saliva was measured by using the spitting method and recorded in $\mathrm{mL} /$ min. Finally, the quantitative gustatory test was conducted 
using the whole mouth chemogustometric method as described in previous literature [13]. Briefly, the test procedure was performed with serial applications of the tastant solutions at 14 different concentration levels in the order of sucrose, sodium chloride, tartaric acid, and quinine hydrochloride. The concentration levels of tastants are shown in Supplementary Table 1 (available online only) [13]. First, $1 \mathrm{~mL}$ aliquot of the lowest concentration (C1) was sprayed from a needle-free syringe over the subject's tongue, who then spitted the solution. The subjects then reported whether or not there was a taste sensation and described the quality of the taste. The same procedure was followed using 1 $\mathrm{mL}$ aliquots of successively higher concentrations of the solution until the subject detected and accurately recognized the taste. The same process was repeated for the other taste solutions. The lowest concentration at which the subject felt a taste sensation was defined as the detection threshold and the lowest concentration at which the subject accurately identified the characteristics of the taste was defined as the recognition threshold [13]. The threshold values were recorded along with the level of taste concentration for each taste quality.

Statistical analysis of the data was performed using SPSS for Windows, Version 12.0 (SPSS Inc., Chicago, IL, USA). Quantitative data were presented as mean \pm standard deviation (SD). The normality test was performed using the Shapiro-Wilk test. For statistical analysis, the KruskalWallis test was applied to compare the differences in detection and recognition thresholds between the hypergeusia, hypogeusia, and dysgeusia groups. Scheffé's test using ranks or Tamhane's T2 using ranks was followed as Post hoc multiple comparisons when appropriate. For statistical comparison between the sex groups, Mann-Whitney U-test was performed to determine the differences in the duration of symptoms and the treatment period; and Chi-square or Fisher's exact test was used to compare the differences in other clinical variables. The significance level was set at $\mathrm{p}<0.05$.

\section{RESULTS}

The hospital records of 169 patients (73 males and 96 females) were analysed retrospectively in this study. The patients' age ranged from the second to the ninth decade (mean age $58.1 \pm 14.5$ years). The age distribution peaked in the 60s (31.4\%) for both men and women, with patients in their 50s (20.7\%) being the next most-affected age group (Fig. 1). In addition, the clinical profiles of patients with taste complaints were analysed together with their sex differences (Table 1). Statistical analyses revealed that there were no significant differences in the sex distribution and age distribution between the sexes.

The mean duration of symptoms after the onset of gustatory dysfunction until the first visit to our clinic was $9.4 \pm 14.2$ (SD) months. A large proportion of the patients (108 patients, 63.9\%) had a history of previous treatments for taste complaints from other clinics such as otolaryngology, local dental clinic, and internal medicine. The average treatment period in our clinic was 65.50 days. However, there were no significant differences in the symptom duration, previous treatment experience, and treatment period between the sexes.

Subjective dry mouth, burning sensation, and tongue coating were the associated symptoms frequently reported in patients with taste complaints. Sixty-one patients (36.1\%) reported subjective oral dryness, which was significantly more common in women (41 patients, 42.7\%) than in men (20 patients, 27.4\%). Furthermore, the salivary flow test revealed that the mean flow rate of unstimulated whole saliva (60.9\%) was $0.2 \mathrm{~mL} / \mathrm{min}$ in 103 subjects and among them, hyposalivation with less than $0.1 \mathrm{~mL} / \mathrm{min}$ was found in 42 subjects (16 males and 26 females). In this study, sixtyfive patients (38.5\%) complained of oral burning sensation,

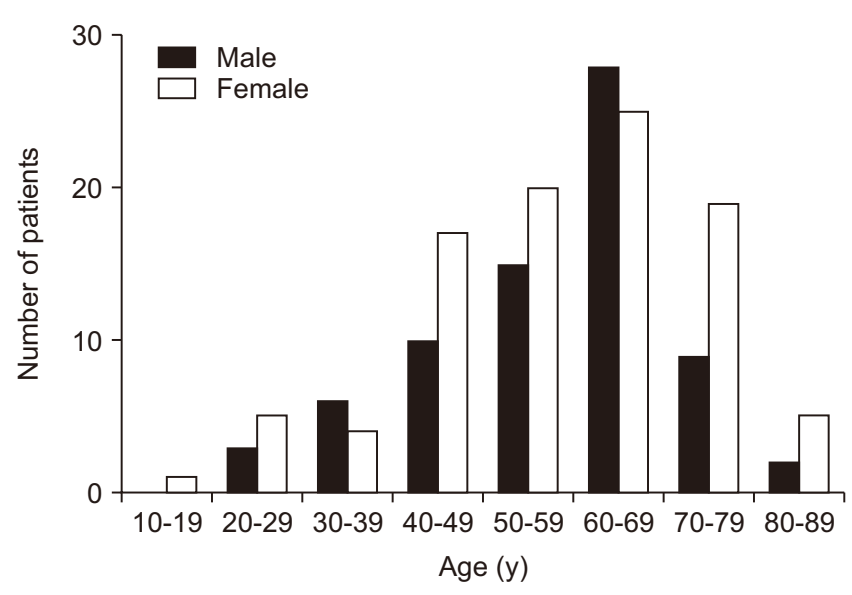

Fig. 1. Age and sex distribution of the subjects. 
Table 1. Distribution of the clinical properties between the sexes

\begin{tabular}{|c|c|c|c|c|}
\hline Variable & Total $(n=169)$ & Male $(n=73)$ & Female $(n=96)$ & $\mathrm{p}$-value \\
\hline Age distribution (y) & & & & $0.759^{\mathrm{a}}$ \\
\hline$\geq 60$ & $88(52.1)$ & $39(53.4)$ & $49(51.0)$ & \\
\hline$<60$ & $81(47.9)$ & $34(46.6)$ & $47(49.0)$ & \\
\hline \multicolumn{5}{|l|}{ Progress } \\
\hline Duration of symptom (m) & $9.36 \pm 14.20$ & $7.75 \pm 12.22$ & $10.69 \pm 15.41$ & $0.103^{b}$ \\
\hline Pre-treatment history & $108(63.9)$ & $45(61.6)$ & $63(65.6)$ & $0.593^{\mathrm{a}}$ \\
\hline Treatment period (d) & $65.50 \pm 194.05$ & $45.10 \pm 167.21$ & $81.01 \pm 209.96$ & $0.317^{b}$ \\
\hline \multicolumn{5}{|l|}{ Associated symptoms } \\
\hline Subjective oral dryness & $61(36.1)$ & $20(27.4)$ & $41(42.7)$ & $0.040 *^{\mathrm{a}}$ \\
\hline Burning mouth sensation & $65(38.5)$ & $20(27.4)$ & $45(46.9)$ & $0.010^{* *^{a}}$ \\
\hline Tongue coating & $43(25.4)$ & $25(34.2)$ & $18(18.8)$ & $0.022^{*^{a}}$ \\
\hline Olfactory dysfunction & $24(14.2)$ & $11(15.1)$ & $13(13.5)$ & $0.778^{\mathrm{a}}$ \\
\hline Changed appetite & $40(23.7)$ & $16(21.9)$ & $24(25.0)$ & $0.641^{\mathrm{a}}$ \\
\hline Oral malodor & $10(5.9)$ & $5(6.8)$ & $5(5.2)$ & $0.747^{c}$ \\
\hline
\end{tabular}

Values are presented as number (\%) or mean \pm standard deviation.

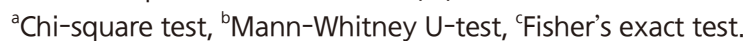

${ }^{*} p<0.05,{ }^{* *} p<0.01$.

Table 2. Systemic conditions of the subjects $(n=169)$

\begin{tabular}{lc}
\hline \multicolumn{1}{c}{ Variable } & Value \\
\hline Current/past medical illness & \\
Allergic disease & $24(14.2)$ \\
Diabetes mellitus & $23(13.6)$ \\
Encephalopathy & $17(10.1)$ \\
Thyroid problem & $13(7.7)$ \\
Gastroesophageal reflux disorder & $11(6.5)$ \\
Hepatic disease & $7(4.1)$ \\
Psychiatric problem & $7(4.1)$ \\
Renal disease & $4(2.4)$ \\
Medication intake status & \\
Over 12 months & $77(45.6)$ \\
\hline
\end{tabular}

Values are presented as number (\%).

which was significantly more common in women (45 patients, 46.9\%) than in men (20 patients, 27.4\%). Oral examination revealed that 43 patients (25.4\%) had an apparent tongue coating, which was significantly more common in men (25 patients, 34.2\%) than in women (18 patients, $18.8 \%)$.

Regarding the coexistence of other chemosensory dysfunction, olfactory disorders were reported only in 24 patients (14.2\%). Forty patients (23.7\%) complained of decreased appetite due to taste abnormality and 10 patients (5.9\%) reported oral malodour or bad breath.

With regard to the general health conditions, the most frequent systemic disease was allergic disease, accounting for 24 patients (14.2\%), followed by diabetes mellitus (23

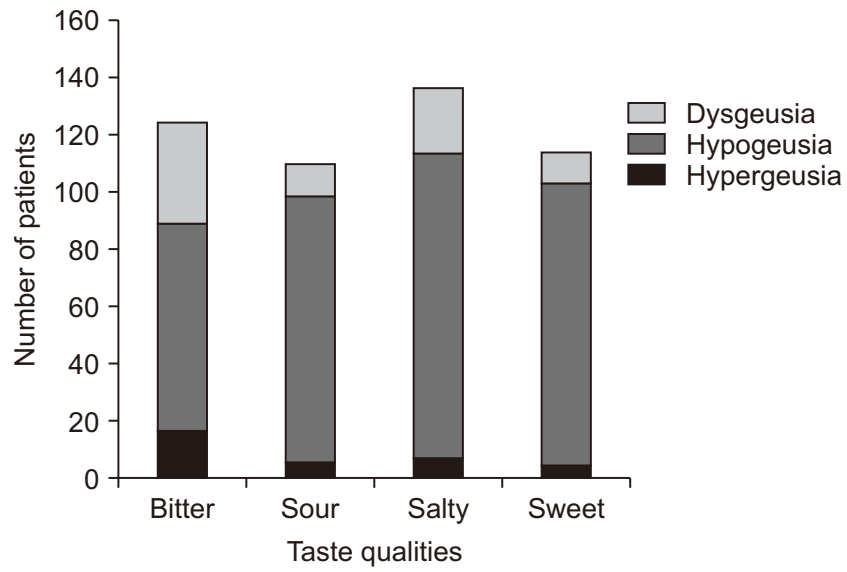

Fig. 2. Distribution of the clinical subtypes of taste complaints.

patients, 13.6\%), and encephalopathy (17 patients, 10.1\%). Of the 169 patients, 77 patients (45.6\%) took more than one medication for more than 12 months. The detailed systemic conditions and medication status are also presented in Table 2.

The distribution of the clinical subtypes of taste dysfunction is presented in Fig. 2. Hypogeusia (76.3\%) was the most common subtype of taste complaints, followed by dysgeusia (16.3\%), and hypergeusia (only 7.4\%). In addition, further comparison of the gustatory thresholds is shown in Fig. 3. For salty taste, the detection thresholds were significantly elevated among subjects in the hypogeusia group compared to those in the dysgeusia group ( $\mathrm{p}=0.036)$. Similarly, the recognition thresholds in the hypogeusia group also displayed significantly higher thresholds compared to those in 
A Bitter

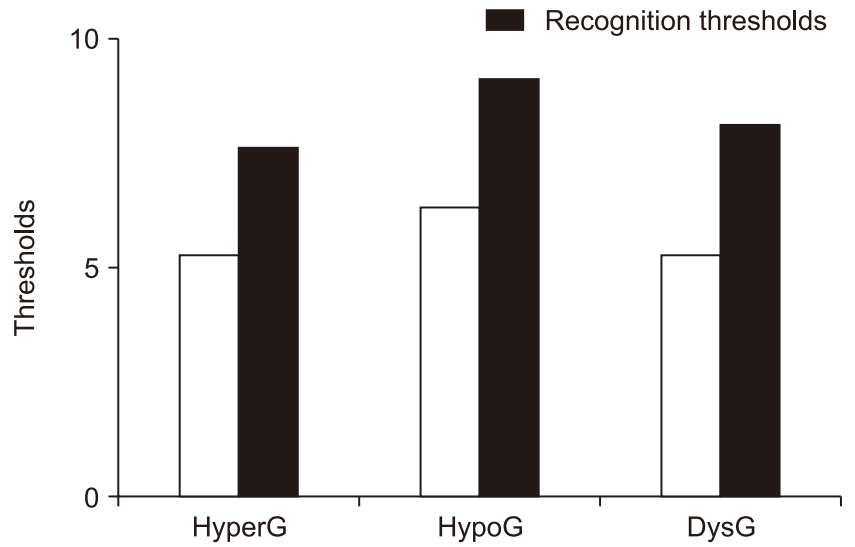

C Salty

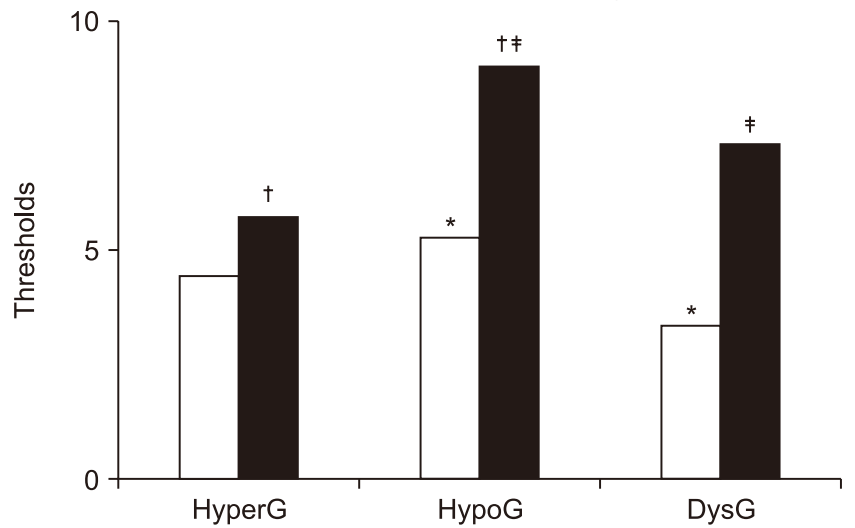

B Sour
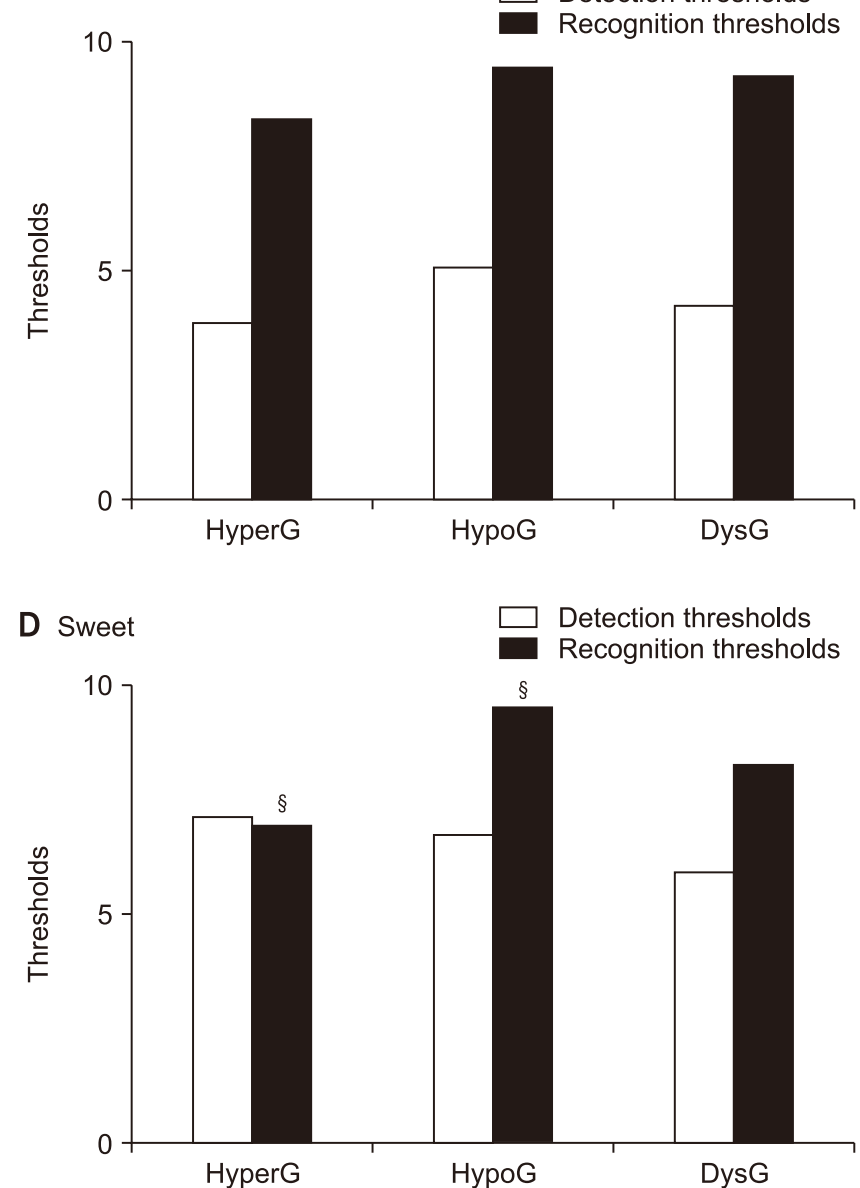

Fig. 3. Comparison of taste thresholds according to the clinical subtypes of taste complaints. (A) Bitter threshold, (B) Sour threshold, (C) Salty threshold, (D) Sweet threshold. HyperG, hypergeusia; HypoG, hypogeusia; DysG, dysgeusia. Scheffé's test using ranks for salty taste, Tamhane's T2 using ranks for sweet taste as Post hoc multiple comparisons. *HypoG vs. DysG in the detection threshold $\left({ }^{*} p<0.05\right)$. ${ }^{\dagger} H y p o G$ vs. HyperG in the recognition threshold $\left({ }^{\dagger} p<0.05\right)$. ${ }^{\ddagger} H y p o G$ vs. Dys in the recognition threshold $\left({ }^{\ddagger} p<0.05\right) .{ }^{8} \mathrm{HypoG}$ vs. HyperG in the recognition threshold $\left({ }^{\S} p<0.01\right)$.

the hypergeusia and dysgeusia groups $(\mathrm{p}=0.033,0.026$, respectively). For sweet taste, there was no significant difference in the detection thresholds between the hypergeusia, hypogeusia, and dysgeusia groups. Statistically significant differences $(p<0.001)$ in the recognition thresholds were also observed between the hypergeusia and hypogeusia groups. For bitter and sour taste, a comparison of the groups showed no statistically significant differences for both detection and recognition thresholds ( $p>0.05$ ).

\section{DISCUSSION}

Taste sensation is essentially defined as the specialized chemosensation in the taste bud that senses the chemical molecules with taste qualities. Taste qualities have been traditionally subdivided into sweet, salty, sour, bitter, and umami, depending on the nature of the taste perceived in the brain. Unlike other special sensations like visual sensation, taste perception is more complicated due to its integration with other sensory inputs before taste perception and discrimination occurs at the cerebral cortex level [5]. Previous literature has indicated that most taste complaints could be caused by disturbances in olfactory sensation and instead of the gustatory sensation itself [14-16]. The complex process of taste perception plays a central role in the clinical diversity of taste complaints in patients, thereby leading to challenges for clinicians. It was therefore recommended that patients with taste complaints should be carefully examined using the quantitative assessment, as well as the clinical evaluation, focused on the taste sensitivity 
and the possible causes $[5,15]$. While several studies reported the diversity in clinical characteristics of patients with taste complaints, there have been fewer reports that analyse the sex differences in the clinical profiles and determine the differential pattern of quantitative gustatory outcome according to the clinical types of taste complaints $[10,15]$.

Regarding the clinical profiles of patients with taste complaints, this study showed that patients in their sixties formed the largest age group (31.4\%), whereas patients under 29 years accounted for only 5.3\%. Several previous studies also indicated age-related changes in taste sensitivity with a progressively increasing prevalence rate with age $[6,9,17]$. Our findings confirmed previous observations regarding the possible effects of age on the taste complaints $[6,18]$. This tendency could be interpreted as a consequence of the increased prevalence of systemic diseases and medications with aging $[6,18,19]$. Furthermore, it was suggested that older individuals were more vulnerable to the same causes of taste dysfunction than the young age group [20]. It was also reported that the loss of taste among the older population was more commonly caused by nutritional deficiency, and oral and systemic diseases [21]. In terms of the sex proportion of the patients with taste complaints, there were no significant differences between the sexes, even though females showed a slightly higher ratio of 1:1.2.

Our study revealed that a considerable proportion of patients (45.6\%) were taking one or more medicines for over 12 months, i.e. antihypertensives, cardiac medications, antidiabetic drugs, thyroid drugs, medications for several types of brain diseases, antidepressants, and gastrointestinal medicines. Drug-induced taste disorders were reported as the most common type of taste disorders [3]. A number of drugs are known to have the potential to disturb the normal taste function by various mechanisms [22]. Drug-induced taste disorders could occur by inducing a neuronal dysfunction mainly through disturbing the ion channels and cellular homeostasis in the taste buds $[23,24]$. The gustatory system can be partly influenced by medications that alter the chemical composition or flow rate of saliva [25]. However, since these mechanisms are not fully understood, it is not easy to determine the direct link between individual drugs taken by the patients and their taste complaints. In addition, systemic conditions, such as endocrine and metabolic disorders have been reported to cause abnormalities in taste sensation $[14,26,27]$. This study found that allergic disease was the most frequent systemic disease followed by diabetes mellitus. It might be implied that antihistamines and decongestants for allergic diseases may affect the taste sensation or probably the olfactory sensation.

In this study, 24 patients (14.2\%) reported subjective olfactory disturbances, while previous literature has described that most self-reported cases of taste loss were confused with olfactory loss due to the limited ability to discriminate complex flavours of food/beverages $[2,5,19,28]$. However, since this prevalence of olfactory loss was based only on questionnaires and interviews instead of quantitative olfactory assessment, it could be potentially under-estimated in this study and may be inadequate to determine the effect of olfactory loss on taste complaints.

Regarding the sex differences in clinical profiles, this study revealed that subjective dry mouth was significantly more common in females than in males with taste complaints, while coated tongue was significantly more frequent in males than in females. Previous studies indicated that dry mouth and tongue coating could cause disturbances in taste sensation through insufficient solution and blocked delivery of the taste components within the food $[2,14,19]$. Our results were consistent with previous findings that xerostomia and hyposalivation were more prevalent in women $[29,30]$. A recent study reported that tongue coating was significantly higher in males and there was a strong correlation between tongue coating and increasing age [31]. Collectively, these findings indicated that the local causes for taste complaints may be different between males and females, subsequently leading to dental clinicians considering more individualized strategies during oral examination and treatment. In addition, our study found that the burning sensations were significantly more common in females than in males with taste complaints. This finding was in accordance with the findings that burning mouth syndrome was more prevalent in the middle-aged female population $[32,33]$. Other studies showed that complaints of burning mouth were more frequent in women (12.3\%) than in men (5.4\%) with gustatory or olfactory complaints [10].

A variety of quantitative tests were clinically and experimentally developed with a focus on gustatory sensitivity 
$[14,26,34]$. To date, there have been several methods to evaluate taste sensitivity, which are mainly classified into electrogustometry and chemogustometry. While electrogustometry was used to quantitatively measure the sensory thresholds of the taste buds in response to electrical stimuli rather than chemical stimuli, chemogustometric tests were performed to assess the gustatory threshold to detect and identify the given taste quality using taste solutions with different concentrations and taste qualities $[12,13,34]$. Although electrogustometry was considered to be more practical due to the relatively simple and short examination procedure, chemogustometric methods involving the application of taste components were considered to be more appropriate in representing the quantitative and qualitative aspects of taste sensations [26,35]. In the present study, the taste thresholds were also determined using one of the most commonly used chemogustometric methods, as described previously [13]. However, despite the numerous studies, there is no definitive range of normal values of taste thresholds due to insufficient samples and diverse outcomes, which makes it hard to determine whether the measured values fall within the normal range [36-38].

In the present study, analysis of the quantitative gustatory outcomes depending on the clinical subtypes of taste disorders showed that the taste thresholds were not always consistent with the patient-described gustatory symptoms. A previous study reported that self-reported questionnaires had low accuracy in detecting taste dysfunctions [15]. Another study based on quantitative gustatory and olfactory tests also revealed that only $25.4 \%$ patients had abnormal gustatory function among the patients with only taste complaints and 9.5\% had an abnormal gustatory function for those reporting taste and smell complaints [5]. In addition, our study showed that for salty and sweet tastes, the gustatory thresholds seemed to correspond to the patientdescribed symptoms, while there were no significant differences between the hypergeusia, hypogeusia, and dysgeusia groups in the taste thresholds for bitter and sour tastes. The reasons for these differences in the quantitative gustatory outcomes are not clear. These wide variations in the taste thresholds of patients with taste complaints might be explained by the dynamic and distributed characteristics of gustatory processing. The gustatory system consists of multiple levels of interacting neural network distribution. Each gustatory nucleus is reciprocally connected, and the outputs of each nucleus may affect the activity of the other. Furthermore, these gustatory nuclei interact with information from the somatosensory system as well $[39,40]$. Subjective taste complaints also appear to be a consequence of the sensory disturbances of psychiatric origin [41]. However, since taste disorders are clinically defined as abnormal chemical senses responding to taste compounds, it is hard to objectively determine the presence and severity of taste disorders. The patient response, even by quantitative analysis, would be subjective and individualized with psychological involvements [12].

Therefore, dental clinicians would inevitably encounter inconsistencies between the patients' subjective taste complaints and the results of the taste threshold tests. Nevertheless, our findings indicate that patients with taste complaints could present not only with true dysfunction in the taste sensation, but also with the other disturbances related with taste perception. The quantitative taste measurements could be clinically meaningful as useful diagnostic tools capable of differentiating true taste dysfunctions among the heterogeneous taste complaints.

There are several intrinsic limitations to the present study. First, due to the retrospective design of this study, there was some missing information in some of the cases. Second, our information did not include sufficient psychiatric conditions affecting chemosensory dysfunction. Third, laboratory tests to evaluate any possible systemic conditions affecting the taste sensations were not performed for all patients. In other words, all the conditions that could impede taste function were not controlled, making it difficult to differentiate the true loss of taste from other chemosensory dysfunctions.

In conclusion, patients with taste complaints had diverse clinical profiles, ranging from true taste sensory dysfunction to disturbed taste perception with normal sensation. Considering the diversity of taste complaints, the quantitative gustatory testing methods are valuable to differentially evaluate the presence and intensity of taste sensation in patients with taste complaints. 


\section{CONFLICT OF INTEREST}

No potential conflict of interest relevant to this article was reported.

\section{ORCID}

\author{
Seo-Yeong Kim \\ https://orcid.org/0000-0002-8555-2490 \\ Jin-Seok Byun \\ https://orcid.org/0000-0002-6182-1238 \\ Jae-Kwang Jung \\ https://orcid.org/0000-0003-3099-8097 \\ Jae-Kap Choi \\ https://orcid.org/0000-0001-6773-7507
}

\section{REFERENCES}

1. Wardle J, Cooke LJ. One man's meat is another man's poison. Science \& Society series on food and science. EMBO Rep 2010;11:816-821.

2. Maheswaran T, Abikshyeet P, Sitra G, Gokulanathan S, Vaithiyanadane V, Jeelani S. Gustatory dysfunction. J Pharm Bioallied Sci 2014;6(Suppl 1):S30-S33.

3. Hamada N, Endo S, Tomita H. Characteristics of 2278 patients visiting the Nihon University Hospital Taste Clinic over a 10-year period with special reference to age and sex distributions. Acta Otolaryngol Suppl 2002;(546):7-15.

4. Ship JA, Chavez EM. Special senses: disorders of taste and smell. In: Silverman S, Eversole LR, Truelove EL, eds. Essentials of oral medicine. Hamilton: BC Decker Inc.; 2001. pp. 279-280.

5. Hunt JD, Reiter ER, Costanzo RM. Etiology of subjective taste loss. Int Forum Allergy Rhinol 2019;9:409-412.

6. Hoffman HJ, Ishii EK, MacTurk RH. Age-related changes in the prevalence of smell/taste problems among the United States adult population. Results of the 1994 disability supplement to the National Health Interview Survey (NHIS). Ann N Y Acad Sci 1998;855:716-722.

7. Bergdahl M, Bergdahl J. Perceived taste disturbance in adults: prevalence and association with oral and psychological factors and medication. Clin Oral Investig 2002;6:145-149.

8. Welge-Lüssen A, Dörig P, Wolfensberger M, Krone F, Hummel T. A study about the frequency of taste disorders. J Neurol 2011; 258:386-392.

9. Rawal S, Hoffman HJ, Bainbridge KE, Huedo-Medina TB, Duffy VB. Prevalence and risk factors of self-reported smell and taste alterations: results from the 2011-2012 US National Health and Nutrition Examination Survey (NHANES). Chem Senses 2016;41:69-76.

10. Deems DA, Doty RL, Settle RG, et al. Smell and taste disorders, a study of 750 patients from the University of Pennsylvania Smell and Taste Center. Arch Otolaryngol Head Neck Surg 1991;117:519-528.

11. Fark T, Hummel C, Hähner A, Nin T, Hummel T. Characteristics of taste disorders. Eur Arch Otorhinolaryngol 2013;270:1855-1860.

12. Naik C, Claussen CF. Qualitative and quantitative representation of taste disturbances: how we do it by pentagon chart. Indian J Otolaryngol Head Neck Surg 2010;62:376-380.

13. Yamauchi Y, Endo S, Sakai F, Yoshimura I. A new whole-mouth gustatory test procedure. 1. Thresholds and principal components analysis in healthy men and women. Acta Otolaryngol Suppl 2002;(546):39-48.

14. Allis TJ, Leopold DA. Smell and taste disorders. Facial Plast Surg Clin North Am 2012;20:93-111.

15. Soter A, Kim J, Jackman A, Tourbier I, Kaul A, Doty RL. Accuracy of self-report in detecting taste dysfunction. Laryngoscope 2008;118:611-617.

16. Malaty J, Malaty IA. Smell and taste disorders in primary care Am Fam Physician 2013;88:852-859.

17. Boesveldt S, Lindau ST, McClintock MK, Hummel T, Lundstrom JN. Gustatory and olfactory dysfunction in older adults: a national probability study. Rhinology 2011;49:324-330.

18. Liu G, Zong G, Doty RL, Sun Q. Prevalence and risk factors of taste and smell impairment in a nationwide representative sample of the US population: a cross-sectional study. BMJ Open 2016;6:e013246.

19. Su N, Ching V, Grushka M. Taste disorders: a review. J Can Dent Assoc 2013;79:d86.

20. Seiberling KA, Conley DB. Aging and olfactory and taste function. Otolaryngol Clin North Am 2004;37:1209-1228, vii.

21. Imoscopi A, Inelmen EM, Sergi G, Miotto F, Manzato E. Taste loss in the elderly: epidemiology, causes and consequences. Aging Clin Exp Res 2012;24:570-579.

22. Doty RL, Shah M, Bromley SM. Drug-induced taste disorders. Drug Saf 2008;31:199-215.

23. Yang L, Xie G, Fan Q, Xie J. Activation of the hedgehog-signaling pathway in human cancer and the clinical implications. Oncogene 2010;29:469-481.

24. Naik BS, Shetty N, Maben EV. Drug-induced taste disorders. Eur J Intern Med 2010;21:240-243.

25. Spielman AI. Chemosensory function and dysfunction. Crit Rev Oral Biol Med 1998;9:267-291.

26. Wrobel BB, Leopold DA. Clinical assessment of patients with smell and taste disorders. Otolaryngol Clin North Am 2004;37: 1127-1142.

27. Doty RL. Gustation. Wiley Interdiscip Rev Cogn Sci 2012;3:2946.

28. Stevenson RJ, Prescott J, Boakes RA. Confusing tastes and smells: how odours can influence the perception of sweet and sour tastes. Chem Senses 1999;24:627-635.

29. Guggenheimer J, Moore PA. Xerostomia: etiology, recognition and treatment. J Am Dent Assoc 2003;134:61-69; quiz 118-119.

30. Ohara Y, Hirano H, Yoshida H, et al. Prevalence and factors associated with xerostomia and hyposalivation among communitydwelling older people in Japan. Gerodontology 2016;33:20-27. 
31. Omor RA, Arabeyat MA, Hiasat AN, Ajarmeh MS, Abu Fanas HH. Prevalence and factors related to tongue coating among a sample of Jordanian royal medical services dental outpatients. JRMS 2015;22:35-40.

32. Aravindhan R, Vidyalakshmi S, Kumar MS, Satheesh C, Balasubramanium AM, Prasad VS. Burning mouth syndrome: a review on its diagnostic and therapeutic approach. J Pharm Bioallied Sci 2014;6(Suppl 1):S21-S25.

33. Feller L, Fourie J, Bouckaert M, Khammissa RAG, Ballyram R, Lemmer J. Burning mouth syndrome: aetiopathogenesis and principles of management. Pain Res Manag 2017. doi:10.1155/2017/1926269.

34. Ambaldhage VK, Puttabuddi JH, Nunsavath PN, Tummuru YR. Taste disorders: a review. J Indian Acad Oral Med Radiol 2014;26:69-76.

35. Ellegård EK, Hay KD, Morton RP. Is electrogustometry useful for screening abnormalities of taste? J Laryngol Otol 2007;121:11611164 .
36. Landis BN, Welge-Luessen A, Brämerson A, et al. "Taste Strips" a rapid, lateralized, gustatory bedside identification test based on impregnated filter papers. J Neurol 2009;256:242-248.

37. Lee JW, Shin SH, Rhyu MR, Kim JY, Ye MK. The effect of aging on taste thresholds in Korean. Korean J Otolaryngol-Head Neck Surg 2013;56:286-290.

38. Son HJ, Lee JW, Shin SH, Rhyu MR, Kim JY, Ye MK. Differences in taste thresholds according to sex and age groups in Korean. Korean J Otolaryngol-Head Neck Surg 2014;57:692-697.

39. Katz DB, Nicolelis MA, Simon SA. Gustatory processing is dynamic and distributed. Curr Opin Neurobiol 2002;12:448-454.

40. Simon SA, de Araujo IE, Stapleton JR, Nicolelis MA. Multisensory processing of gustatory stimuli. Chemosens Percept 2008;1:95102.

41. Osaki T, Ohshima M, Tomita Y, Matsugi N, Nomura Y. Clinical and physiological investigations in patients with taste abnormality. J Oral Pathol Med 1996;25:38-43. 